\title{
Supplementation of $\Omega-3$ Fatty Acids in Parenteral Nutrition Beneficially Alters Phospholipid Fatty Acid Pattern
}

\author{
Metin Senkal, MD*; Bruno Geier, MD*; Martin Hannemann, MD*; Thomas Deska, MD*; \\ Jakob Linseisen, PhD†; Günther Wolfram, MD ; and Michael Adolph, MD§
}

\begin{abstract}
From the *Department of Surgery, Ruhr-University Bochum, St. Josef Hospital, Bochum, Germany; †German Cancer Research Center, Heidelberg, Germany; $¥ D e p a r t m e n t$ of Nutrition and Food Sciences, Technical University Munich, Freising, Germany; and the §Department of Anesthesiology and Intensive Care Medicine, Tuebingen University Hospital, Tuebingen, Germany
\end{abstract}

\begin{abstract}
Background: The clinical safety and the uptake of $\omega-3$ polyunsaturated fatty acids (PUFA) into the serum phospholipids and erythrocyte membranes after administration of fish-oil-supplemented parenteral nutrition (PN) was investigated in colorectal surgical patients. Methods: Forty patients undergoing colorectal surgery $(n=40)$ and with an indication for PN were enrolled in a prospective, doubleblind, randomized study to receive an $\omega-3$ PUFA-supplemented 20\% lipid emulsion (Lipoplus; B. Braun Melsungen, Melsungen, Germany; test group, $\mathrm{n}=19$ ) for 5 days postoperatively. The control group received a standard $20 \%$ fat emulsion (Lipofundin MCT/LCT, B. Braun Melsungen, Melsungen, Germany, control group, $\mathrm{n}=21$ ). Clinical outcome parameters and safety were assessed by means of adverse events recording clinical parameters and hematologic analyses. The contents of eicosapentaenoic acid (EPA) and docosahexaenoic acid (DHA), as well as arachidonic acid (AA), in phospholipid fractions in plasma and in erythrocytes were analyzed preoperatively, on postoperative days 1,6 , and 10 using liquid gas chromatography. Results: Both fat emulsions were well tolerated, and none of the adverse events was considered to be related to treatment. Postoperative infectious complications occurred in 4 patients of the $\omega-3$ PUFA group vs 7 patients in the control group. As compared with the control group, the $\omega-3$ PUFA group had significantly
\end{abstract}

The dietary fatty acids of the $\omega-3$ series are rapidly incorporated into cell membranes and profoundly influence biologic responses. ${ }^{1,2}$ These lipids influence membrane stability, membrane fluidity, cell mobility, the formation of receptors, binding of ligands to their receptors, activation of intracellular signaling pathways either directly or through the formation of eicosanoids, gene expression, and cell differentiation. ${ }^{1,3-5}$ In general, eicosanoids formed from the $\omega-3$ fatty acids cause biologic responses opposite to those of the $\omega-6$ fatty acids, including lesser inflammatory responses after trauma or surgery.

Correspondence: Metin Senkal, MD, Department of Surgery, Marienhospital Witten, Marienplatz 2, 58452 Witten, Germany. Electronic mail may be sent to Senkal@Marien-Hospital-Witten.de. increased levels of EPA in the membranes of the erythrocytes in postoperative day $6(2.0 \% \pm 0.9 \%$ vs $0.8 \% \pm 0.5 \%$ fatty acid methyl esters, [FAME]) and postoperative day $10(2.1 \% \pm$ $0.8 \%$ vs $0.9 \% \pm 0.7 \%$ FAME, $p<.05$ ). Also, the EPA levels in the serum phospholipids were significantly higher than in the control group on the same postoperative days $(7.0 \% \pm$ $2.6 \%$ vs $1.3 \% \pm 0.8 \%$ and $3.6 \% \pm 1.0 \%$ vs $1.0 \% \pm 0.4 \%$ FAME, $p<.05)$. The DHA levels in the serum phospholipids were significantly higher in the $\omega-3$ PUFA group compared with the control on postoperative days 6 and $10(11.8 \% \pm 1.9 \%$ vs $8.4 \% \pm 1.5 \%$ and $11.2 \% \pm 1.6 \%$ vs $8.5 \% \pm 1.4 \%$ FAME, $p<$ $.05)$. AA levels were not significantly different in the both groups. Conclusions: $\Omega$-3-fatty-acids-supplemented fat emulsions for parenteral administration are safe and very well tolerated. This study demonstrates that parenteral administration of $\omega$-3-PUFA-enriched fat emulsions leads to increased incorporation of EPA and DHA into phospholipids in serum and erythrocytes, whereas AA levels remain unchanged. Thus, postoperative parenteral administration of $\omega$-3-PUFA-enriched lipid emulsions could have an impact on the postoperative inflammatory response after abdominal surgery and could be used in standard postoperative care when $\mathrm{PN}$ is indicated.

It is now widely accepted that long-chain polyunsaturated fatty acids are not only used as energy-providing substrates but that they possess important additional functions as building blocks for cell membranes and precursors of eicosanoids and cytokines. ${ }^{6}$ Most important, the ratio of $\omega-6$ to $\omega-3$ fatty acids bound in cell membrane phospholipids alters eicosanoid synthesis: a low ratio (high percentage of $\omega-3$ ) can markedly suppress generation of proinflammatory leukotrienes. ${ }^{2,7}$ At least a part of the inflammation exacerbation postsurgery is due to generation of proinflammatory mediators such as leukotrienes and prostaglandins. ,-6 fatty acids serve as precursors of inflammatory mediators associated with injury and the stress response. ${ }^{8}$ In contrast, the antiinflammatory effects of $\omega-3$ polyunsaturated fatty acids (PUFA) have been well documented in both animals and humans. The antiinflammatory properties of $\omega-3$ PUFA have also been demonstrated in injured animals, as manifested by 
reductions in metabolic rates, normalization of glucose metabolism, overall weight loss, and nitrogen balance and immune function. ${ }^{9,10}$ It can be, thus, hypothesized that any nutrition support beneficially modifying cell membrane composition may influence the course and the magnitude of the sequences of proinflammatory cascades after trauma.

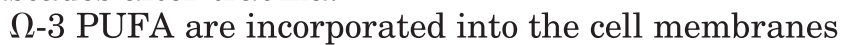
of immune cells-particularly neutrophils, lymphocytes, monocytes, and macrophages-as early as 3 hours after intravenous (IV) infusion. ${ }^{11,12}$ They can displace $\omega-6$ fatty acid derivatives from the membranes of these cells and thereby apparently produce immune system/immune response modulation. ${ }^{13,14}$

Recent research suggests that supplementation of $\omega-3$ fatty acids may have a favorable impact on the length of hospital stay and mortality of surgical and septic patients. ${ }^{6}$

This phase II clinical trial evaluated the incorporation of eicosapentaenoic acid (EPA), docosahexaenoic acid (DHA), and arachidonic acid (AA) into the phospholipids in the serum and in membranes of erythrocytes, as well as the safety/tolerability of a parenteral fat emulsion supplemented with $\omega-3$ fatty acids. The study was designed at a time when parenteral nutrition (PN) was still prescribed for most patients undergoing colorectal surgery. Although the need for PN in normal-weight patients after colorectal surgery may be viewed critically, the homogeneity of such a patient population is ideally suited for a parallel-group comparison of the clinical outcome, safety, and tolerance parameters after parenteral administration of $\omega-3$ fatty acids.

\section{MATERIALS AND METHODS}

Designed as a single-site, prospective, double-blind, parallel-group comparison, the study was conducted in accordance with the recommendations of Good Clinical Practice for Trials on Medicinal Products in the European Community (III/3976/88-EN, final version, July $11,1990)$ and the Declaration of Helsinki (Hong Kong, September 1989) after the protocol had been approved by the Ethics Committee of Ruhr-Universität Bochum, Germany.

\section{Patients}

This study was performed with 40 patients who had been admitted to the Department of General Surgery, St. Josef-Hospital, Bochum, Germany. The patient characteristics are shown in Table I.

Only patients were included who received $\mathrm{PN}$ for at least 5 consecutive days after elective colorectal surgery. Patients were to be between 40 and 80 years of age and had to give informed consent before entering the study.

Patients who did not give informed consent, who participated in another trial, who were pregnant or lactating, who were obese or underweight, and patients with known alcohol or drug abuse were excluded from participation. Patients with dyslipoproteinemia, insulin-dependent diabetes mellitus, hemorrhagic diathesis, known food allergies or other allergic predisposi-
TABLE I

Patient data (mean $\pm S D)$

\begin{tabular}{lcc}
\hline & $\begin{array}{c}\omega-3 \text { PUFA group } \\
(\mathrm{n}=19)\end{array}$ & $\begin{array}{c}\text { Control group } \\
(\mathrm{n}=21)\end{array}$ \\
\hline Gender (male:female) & $10: 9$ & $10: 11$ \\
Mean age (y) & $69 \pm 9.1$ & $66.3 \pm 8.1$ \\
Nutrition status & 1 & 5 \\
$\quad$ Moderate, reduced & 17 & 11 \\
$\quad$ Normal & 1 & 4 \\
$\quad$ Obese & 7 & 4 \\
Surgical procedure & 1 & 1 \\
$\quad$ Right hemicolectomy & 2 & 2 \\
Resection of transverse colon & 3 & 5 \\
$\quad$ Left hemicolectomy & 6 & 9 \\
$\quad$ Resection of anterior rectum & & \\
$\quad$ Other (colon segment resection, & & \\
\hline
\end{tabular}

The nutrition status was evaluated by subjective global assessment (SGA). $\omega-3$ PUFA, polyunsaturated fatty acids.

tion, severe hepatic dysfunction, renal failure, sepsis, congestive heart failure, emergency procedure or lack of adequate preparation for surgery, radio and/or chemotherapy within 6 months of enrollment, other immunosuppressive treatments, or drug therapy with inhibitors of prostaglandin synthesis, such as aspirin or indomethacin, within 2 weeks of the start of the clinical trial were also excluded.

The patients were randomized using the Rancode +3.1 software (IDV, Munich, Germany), and given consecutive patient numbers as they entered the study.

\section{Study Procedures}

Patients were observed from the day before surgery until postoperative day 10 or the day they were discharged. The total duration of the study was 10 months.

The comparative study was started once the diagnosis was made and the need for PN was established. PN was started on day 1 after surgery. Blood and urine samples were collected at the same sampling time points (approximately $7 \mathrm{AM}$ ) before and on days 1, 6, and 8-10 after surgery. Safety was monitored daily during the observation period; safety evaluations included daily recordings of vital signs (heart rate, blood pressure, respiratory rate, body temperature), adverse events and infectious complications, as well as standard safety laboratory parameters.

Standard safety laboratory parameters (including hematology and clinical chemistry) were analyzed using validated methods at the laboratory of St. Josef Hospital in Bochum.

Fatty acid profiles were determined using liquid gas chromatography. The lipids from serum samples and homogenized tissue samples were extracted using chloroform/methanol (2:1; vol:vol). Phospholipids of serum and tissue samples were separated by thin layer chromatography using petroleum ether/ethylmethylketone/acetic acid (84:15:1; vol:vol:vol) as a solvent. Fatty acid methyl esters were obtained by transesterification with trimethysulphonium hydroxide and separated by use of a CP-Sil-99 (Chrompack, Frankfurt, Germany) capillary column, installed in an HP 5890 
TABLE II

Composition of lipid emulsions in the 2 treatment groups (per $1000 \mathrm{~mL}$ of emulsion)

\begin{tabular}{lcc}
\hline & $\omega$-3 PUFA emulsion & Control emulsion \\
\hline Medium-chain triglycerides & $100.0 \mathrm{~g}$ & $100.0 \mathrm{~g}$ \\
Soybean oil & $60.0 \mathrm{~g}$ & $100.0 \mathrm{~g}$ \\
Fractionated fish oil & $40.0 \mathrm{~g}$ & - \\
Egg yolk phospholipids & $12.0 \mathrm{~g}$ & $12.0 \mathrm{~g}$ \\
Glycerol & $25.0 \mathrm{~g}$ & $25.0 \mathrm{~g}$ \\
\hline
\end{tabular}

Daily fat infusion: first and second postoperative day, $0.7 \mathrm{~g}$ fat $/ \mathrm{kg}$ body weight; third to fifth postoperative day, $1.4 \mathrm{~g}$ fat $/ \mathrm{kg}$ body weight. $\omega-3$ PUFA, polyunsaturated fatty acids.

gas chromatograph with flame ionization detector (Hewlett Packard, Taufkirchen, Germany). For separation and identification of the fatty acid methyl ester peaks, standard analytical grade reagents were used.

\section{Nutrition Interventions}

All infusion products were manufactured and supplied by B. Braun Melsungen (Melsungen, Germany), which was also responsible for blinding the 2 different study fat emulsions.

The PN regimen consisted of carbohydrates, amino acids, and lipids administered according to a predefined dosing schedule. The test and control lipid emulsions were filled in 500-mL infusion bottles by $\mathrm{B}$. Braun Melsungen and stored below $25^{\circ} \mathrm{C}$ in a frost-free place.

The test product was a fat emulsion supplemented with $\omega-3$ fatty acids (Lipoplus) and manufactured by B. Braun Melsungen, in exactly the same way as the control product (Lipofundin MCT/LCT 20\%) in terms of manufacturing processes, in-process controls, and control of the finished product (as specified in Sec. 5 of the German Statutory Instrument Regulating Pharmaceutical Manufacturing Plant Operation [PharmBetrV]). The total fat concentration was $20 \%$ (Table II).

The lipid emulsion was infused intravenously at a constant (pump-controlled) rate for 24 hours a day. The patients received $50 \mathrm{~g}$ fat per day, equivalent to $250 \mathrm{~mL}$ of a $20 \%$ fat emulsion on postoperative days 1 and 2 , and $100 \mathrm{~g}$ of fat per day, equivalent to $500 \mathrm{~mL}$ of a $20 \%$ fat emulsion, on days $3-5$. The $\mathrm{PN}$ regimen also included a $10 \%$ amino acid solution (Aminoplasmal $10 \% \mathrm{E})$ and a $20 \%$ glucose solution, which were simultaneously administered by infusion pumps for 24 hours/day. The total caloric intake was $4600 \mathrm{~kJ}$ on postoperative day $1,6800 \mathrm{~kJ}$ on day 2 , and $9600 \mathrm{~kJ}$ on days $3-5$. No enteral or oral food intake (other than caffeine-free, alcohol-free, and sugar-free beverages) was permitted in the first 5 postoperative days.

\section{Outcome Measures}

The primary outcome measures were defined as the fatty acid pattern of EPA, DHA, and AA in serum phospholipids, as well as in the phospholipids of erythrocyte membranes.

Safety was assessed by means of adverse event recording, vital signs (heart rate, blood pressure, res-
TABLE III

Infections in the 2 treatment groups, indicating the start and end (postoperative day) of treatment of the complication

\begin{tabular}{|c|c|c|c|c|}
\hline \multirow{2}{*}{ Infection } & \multicolumn{2}{|c|}{$\omega-3$ PUFA group } & \multicolumn{2}{|c|}{ Control group } \\
\hline & $\mathrm{n}$ & Postop days & $\mathrm{n}$ & Postop days \\
\hline Urinary tract infection & 1 & $10-13$ & 2 & $1-6$ \\
\hline Impaired wound healing & 2 & $\begin{array}{r}9-15 \\
11-21\end{array}$ & 2 & $\begin{array}{l}6-8 \\
7-21 \\
9-20\end{array}$ \\
\hline Wound abscess & 0 & - & 1 & $7-18$ \\
\hline Intraabdominal abscess & 1 & $11-15$ & 0 & - \\
\hline Pneumonia & 0 & - & 1 & $1-8$ \\
\hline Septicemia & 0 & - & 1 & $5-7$ \\
\hline Total & 4 & & 7 & \\
\hline
\end{tabular}

$\omega-3$ PUFA, polyunsaturated fatty acids.

piration rate, body temperature), hematology laboratory, serum chemistry, and urine chemistry.

\section{Statistical Methods}

The results of this study were evaluated exploratively and descriptively. Wilcoxon tests were performed for group comparisons. Nominal data were analyzed using the appropriate contingency table tests. The $p$ values in all tests are two-tailed and were rounded off to 4 decimal places. $p$ Values $<.05$ were considered statistically significant.

\section{RESULTS}

\section{Patients}

A total of 42 patients were included; 2 patients withdrew their informed consent before the start of study treatment, so that the study was performed with 40 patients, with 19 participants receiving the fish-oilsupplemented fat emulsion ( $\omega-3$ PUFA group), and 21 participants receiving Lipofundin MCT/LCT 20\% (control group). The distribution of age, gender, nutrition status, and the types of surgeries were comparable among the 2 treatment groups (Table I). Nutrition status was calculated by subjective global assessment (SGA). The 2 treatment groups showed no differences in vital signs, and the PN was very well tolerated. Also, there was no statistically significant difference in the occurrence of postoperative infectious complications (Table III). A total of 11 complication events were recorded during treatment. Of these, 4 occurred in the test group and 7 occurred in the control group. Two serious adverse events were reported in the control group (pneumonia, central venous catheter septicemia), but both were not considered to be related to treatment. In contrast to the other complications, the onset of the pneumonia and septicemia in the control group occurred during the PN application period.

\section{Lipid Profiles}

The lipid profiles determined in this study included the $\omega-3$ fatty acids EPA and DHA, and the $\omega-6$ fatty acid AA concentrations in serum, as well as in the membranes of erythrocytes. 

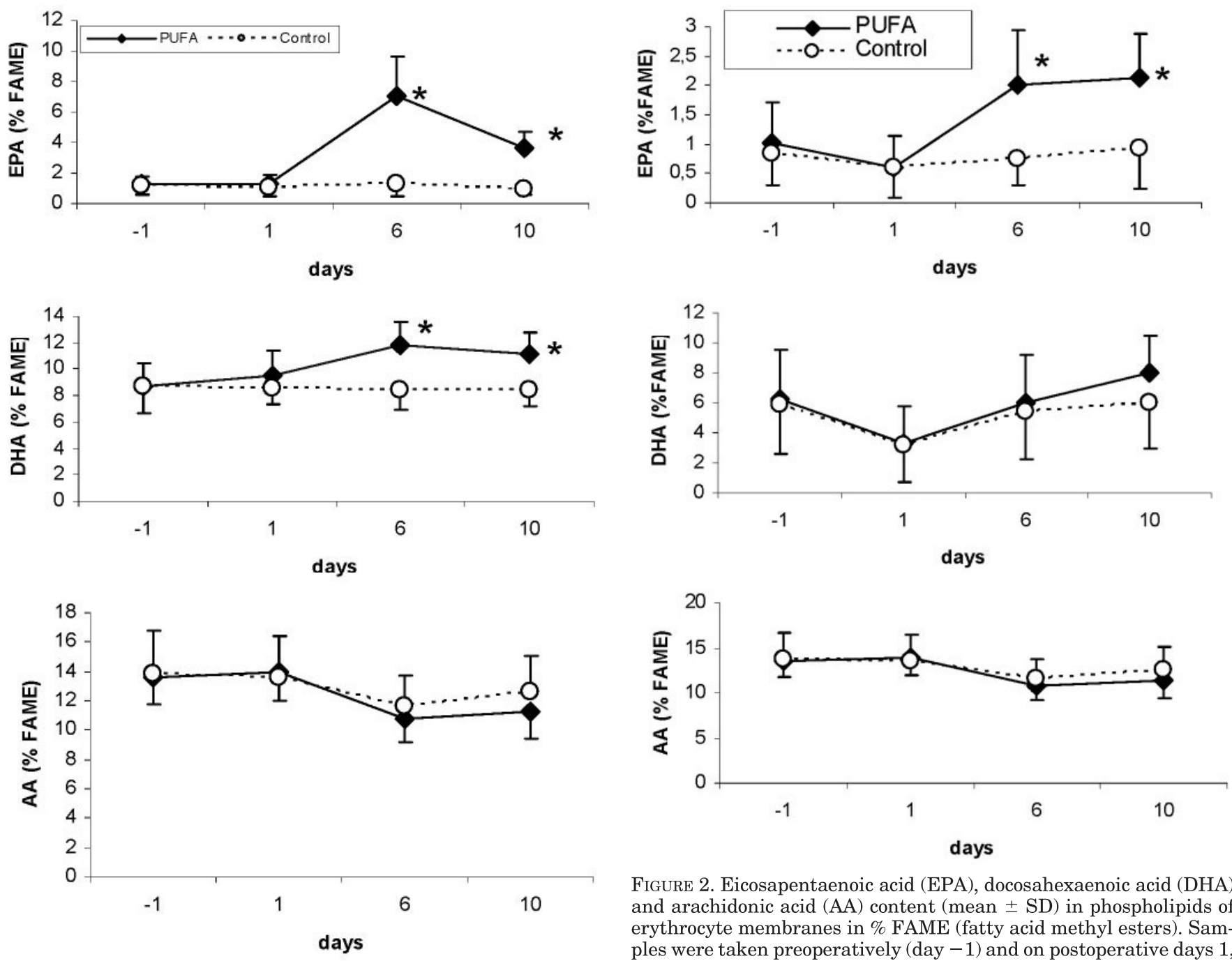

FigURE 2. Eicosapentaenoic acid (EPA), docosahexaenoic acid (DHA) and arachidonic acid (AA) content (mean $\pm \mathrm{SD}$ ) in phospholipids of erythrocyte membranes in \% FAME (fatty acid methyl esters). Samples were taken preoperatively (day -1$)$ and on postoperative days 1 , 6 , and 10 in the group receiving $\omega$-3-fatty-acids-supplemented diet ( $\omega-3$ PUFA, black rhomboids) vs the control group (blank circles) determined by liquid gas chromatography. EPA content was significantly (*) higher in the $\omega-3$ PUFA group on postoperative days 6 and 10 compared with the control group $(p<.05)$. and arachidonic acid (AA) content (mean $\pm \mathrm{SD}$ ) in phospholipids of the serum samples in \% FAME (fatty acid methyl ester) taken preoperatively (day -1$)$ and on postoperative days 1,6 , and 10 in the group receiving $\omega$-3-fatty-acids-supplemented diet ( $\omega-3$ PUFA, black rhomboids) vs the control group (blank circles) determined by liquid gas chromatography. EPA and DHA content was significantly (*) higher in the $\omega-3$ PUFA group on postoperative days 6 and 10 compared with the control group $(p<.05)$.

Figure 1 demonstrates serum EPA and DHA, as well as AA concentrations in both study groups. After 5 days of postoperative PN, there was a significant $(p<$ .05 ) increase in EPA and DHA content in serum phospholipids (postoperative days 6 and 10) under PUFAsupplemented fat emulsion, but not after nutrition support with the reference emulsion. These differences in EPA and DHA uptake into the serum phospholipids between both treatment groups were statistically significant compared with the preoperative day $1(p<.01)$ and postoperative day $1(p<.01)$. Serum AA concentrations decreased significantly in both groups from day 1 to day 6 , with a more pronounced decrease in the $\omega-3$ PUFA group, but without statistically significant difference compared with the control group. Consequently, the $\omega-3: \omega-6$ fatty acid ratio was significantly

higher on postoperative days 6 and 10 in the supplemented patient group (data not shown).

EPA, DHA, and AA content in erythrocyte membranes are shown in Figure 2. Only EPA was significantly $(p<.05)$ increased on postoperative days 6 and 10 in the PUFA group compared with the control group, whereas DHA and AA levels did not show any statistically significant values between both treatment groups. Thus, the ratio of EPA to AA was significantly higher in the $\omega-3$ PUFA group (data not shown).

\section{DISCUSSION}

The aim of the present study was to evaluate changes in lipid composition of serum phospholipids and erythrocyte membranes following PN with $\omega-3$ PUFA supplemented fat emulsions. A secondary aim was to evaluate the safety and tolerance of these emulsions in the clinical setting. In this prospectively randomized, double-blind, parallel-group comparison study, we demonstrated that 5 days of postoperative 
parenteral administration of a $\omega$-3-PUFA-enriched fat emulsion was clinically well tolerated. This treatment significantly increased the content of EPA and DHA in serum phospholipids and erythrocyte membranes. In view of the AA-antagonist role of EPA in inflammatory mediators,${ }^{15,16}$ the fatty acid profiles were of special interest. Serum, as well as erythrocyte, EPA and AA concentrations were determined. As expected, the $\omega-3$ fatty acid concentrations increased significantly during treatment in serum (EPA and DHA), as well as erythrocytes (EPA), in the patients treated with fish oil, but no significant change was observed for the control group. On the other hand, serum AA concentrations decreased significantly in both groups, but the decrease in the treatment group was higher than in the control group. Erythrocyte AA concentrations also decreased during treatment in both groups, but no differences between groups were found. Accordingly, the serum and erythrocyte EPA:AA ratios increased significantly during treatment in the fish-oil-supplemented group, but no changes were observed in the control group (data not shown).

This is also the first phase II clinical trial investigating the safety, as well as potential effects, of a fish-oilsupplemented parenteral fat emulsion (4\% fractionated fish oil $\approx 2-4 \mathrm{~g} / \mathrm{d}$ ) in patients with a defined trauma (colorectal surgery). The test emulsion contained a standardized 5:3:2 ratio of MCT:LCT:fish oil. The control fat emulsion was a commercially available fat emulsion with an MCT:LCT ratio of 1:1 (Lipofundin MCT/LCT 20\%), in contrast to all other studies, where only LCT-based fat emulsions were used. Parenteral fat met $30 \%$ of the total daily energy requirements. The patients received $0.7 \mathrm{~g} / \mathrm{kg}$ of fat on the first 2 postoperative days and $1.4 \mathrm{~g} / \mathrm{kg}$ on postoperative days 3 through 5; the $\omega-6: \omega-3$ fatty acid ratio was $1.5: 1$. This body-weight-related dosage of a standardized, continuously infused lipid emulsion complied with recommendations in the literature. ${ }^{14,17,18}$ According to an average energy requirement of approximately 130 $\mathrm{kJ} / \mathrm{kg} / \mathrm{d}$, both PN regimens had an identical energy content from postoperative day 3 through postoperative day $5 .^{18,19}$ The carbohydrate/fat ratio was $1: 1$ in both treatment groups.

Only 11 postoperative infectious complications were reported during the study in all patients, and none of them was considered to be treatment-related. Although the incidence of postoperative complication was lower in the $\omega-3$ PUFA-supplemented group compared with the control group $(\mathrm{n}=4 v s \mathrm{n}=7)$, this did not reach statistical significance. There were no other adverse events due to the administration of fat emulsions in both groups. No side effects were attributable to the test product and no allergic reactions were observed.

Application of $\omega-3$ fatty acids was demonstrated in various animal models, as well as in humans, to improve host defense mechanisms, ${ }^{20,21}$ and despite some concerns about a further suppression of cell-mediated immune mechanisms in immune-compromised patients, fish oils have been used successfully in the management of several inflammatory, cardiovascular, and autoimmune diseases. ${ }^{6,22-24}$ In vivo and in vitro studies have shown that $\omega-3$ fatty acid-supplemented diets can induce immunomodulation. . $^{5,16,25,26}$

AA and EPA are incorporated into leukocyte phospholipids under dietary conditions. ${ }^{27}$ The enzymes that produce proinflammatory lipid mediators $\left(\mathrm{eg}, \mathrm{LTB}_{4}\right)$ from AA also metabolize EPA and DHA to less inflammatory active mediators like $\mathrm{LTB}_{5}$. Therefore, higher levels of EPA and DHA may lead to a decrease in $\mathrm{LTB}_{4}$ generation, with higher levels of less biologically active mediators such as $\mathrm{LTB}_{5}$. This increase in $\mathrm{LTB}_{5}$ generation with a change in $\mathrm{LTB}_{4}: \mathrm{LTB}_{5}$ ratio was shown in a study where patients were fed with an immune modulating diet preoperatively. ${ }^{28}$

According to a hypothesis advanced by Kinsella et $\mathrm{al}^{14}$ in 1990, Grimm et $\mathrm{al}^{18}$ showed that IV lipid emulsions have different immune-modulating activities, depending on their $\omega-3 / \omega-6$ fatty acid ratio, in a standardized immunostimulation model. This observation may be explained by the fact that the synthetic pathways of $\omega-3$ and $\omega-6$ derivatives compete for the same enzyme system, the desaturase-elongase complex, which shows a preference for $\omega-3$ fatty acids, even causing AA to be displaced from the cell membrane. ${ }^{29,30}$ This means that the $\omega-3$ derivative EPA may act as an AA antagonist, leading to alterations in lipid mediators. ${ }^{31,32}$ In the context of the roles of $\omega-3$ fatty acids in inflammation and coagulation, patients may benefit from $\omega-3$ supplementation of parenteral lipid emulsions.

Interestingly, fish oil supplementation, including a short preoperative parenteral administration of $\omega-3$ fatty acids, significantly reduced mortality rates. ${ }^{12}$ This is explained by beneficial effects of modulation of eicosanoid and cytokine biology. IV infusion of fish oil rapidly leads to incorporation of $\omega-3$ fatty acids in leukocyte cell membrane phospholipids in healthy adults, ${ }^{33,34}$ as well as in different patient groups. ${ }^{728,35-38}$ Infusion of EPA counteracts the fall of cell-mediated immunity after chemoradiation therapy in esophageal cancer patients, as outlined by natural killer cell activity and lymphocyte proliferation. ${ }^{39} \mathrm{~A}$ higher ratio of $\omega-3$ to $\omega-6$ fatty acids in membrane phospholipids is associated with a reduced production of proinflammatory cytokines like IL- $1 \alpha$ and IL-1 $\beta$, IL-6, as well as TNF- $\alpha$, in response to an inflammatory stimulus. $^{40,41}$

In summary, the study shows clearly that postoperative PN with $\omega-3$ fatty acid-supplemented fat emulsion is clinically safe and very well tolerated. Also, immediate administration of fish-oil-supplemented fat emulsion will change the lipid composition of the serum phospholipids and lipids in the erythrocyte membranes, which is a precondition to shift the production of inflammatory mediators toward leukotrienes with less inflammatory potential. Thereby, these data support the hypothesis that alteration of cell membrane phospholipids with active precursors is possible by parenteral administration of $\omega-3$ fatty acids. Due to a decrease in generation of active lipid metabolites from AA, the immune depression following surgical trauma might be diminished. The use of $\omega-3$ PUFA-supplemented fat emulsions must therefore be reevaluated on the background of immune-modulation, 
which might have a beneficial effect on the postoperative course. Because $\omega-3$ PUFA-supplemented parenteral fat emulsions are clinically safe and well tolerated, their use in the clinical setting might be beneficial in patients where immune modulation toward less inflammation reaction is desired.

\section{ACKNOWLEDGMENTS}

The study was supported in part by B. Braun Melsungen AG, Melsungen, Germany.

\section{REFERENCES}

1. Grimble R. Use of n-3 fatty acid-containing lipid emulsions in the intensive care unit environment: the scientist's view. Clin Nutr. 2002;21(suppl 2):15-21.

2. Grimm H, Mayer K, Mayser P, Eigenbrodt E. Regulatory potential of n-3 fatty acids in immunological and inflammatory processes. Br J Nutr. 2002;87(suppl):59-67.

3. Grimminger F, Seeger W, Mayer K. Use of n-3 fatty acid-containing lipid emulsions in the intensive care unit environment: the clinician's view. Clin Nutr. 2002;21(suppl 2):23-29.

4. Koch T, Heller AR. Effects of intravenous fish oil on pulmonary integrity and function. Clin Nutr. 2002;21(suppl 2):41-45.

5. Pscheidl EM. Effects of n-3 fatty acids on gut integrity and function. Clin Nutr. 2002;21(suppl 2):47-51.

6. Furst P, Kuhn KS. Fish oil emulsions: what benefits can they bring? Clin Nutr. 2000;19:7-14.

7. Köller M, Senkal M, Kemen M, et al. Impact of omega-3 fatty acid enriched TPN on leukotriene synthesis by leukocytes after major surgery. Clin Nutr. 2003;22:59-64.

8. Kinsella JE, Lokesh B. Dietary lipids, eicosanoids and the immune system. Crit Care Med. 1999;18:94-113.

9. Sierra P, Ling PR, Istfan NW, Bistrian BR. Fish oil feeding improves muscle glucose uptake in tumor necrosis factor-treated rats. Metabolism. 1995;44:1365-1370.

10. Trocki O, Heyd TJ, Waymack JP, Alexander JW. Effects of fish oil on postburn metabolism and immunity. JPEN $J$ Parenter Enteral Nutr. 1987;11:521-528.

11. Breil I, Koch T, Heller A, et al. Alteration of n-3 fatty acid composition in lung tissue after short-term infusion of fish oil emulsion attenuates inflammatory vascular reaction. Crit Care Med. 1996;24:1893-1902.

12. Tsekos E, Reuter C, Stehle P, Boeden G. Perioperative administration of parenteral fish oil supplements in a routine clinical setting improves patient outcome after major abdominal surgery. Clin Nutr. 2004;23:325-330.

13. Gogos CA, Ginopoulos P, Salsa B, Apostolidou E, Zoumbos NC, Kalfarentzos F. Dietary omega-3 polyunsaturated fatty acids plus vitamin E restore immunodeficiency and prolong survival for severely ill patients with generalized malignancy: a randomized control trial. Cancer. 1998;82:395-402.

14. Kinsella JE, Lokesh B, Broughton S, Whelan J. Dietary polyunsaturated fatty acids and eicosanoids: potential effects on the modulation of inflammatory and immune cells: an overview. Nutrition. 1990;6:24-40.

15. Calder PC. n-3 Polyunsaturated fatty acids and cytokine production in health and disease. Ann Nutr Metab. 1997;41:203-234.

16. Calder PC. Polyunsaturated fatty acids, inflammation, and immunity. Lipids. 2001;36:1007-1024.

17. Galli C, Marangoni F. Recent advances in the biology of n-6 fatty acids. Nutrition. 1997;13:978-985.

18. Grimm H, Tibell A, Norrlind B, et al. Immunoregulation by parenteral lipids: impact of the n-3 to n-6 fatty acid ratio. JPEN J Parenter Enteral Nutr. 1994;18:417-421.

19. Adolph M, Hailer S, Eckart J. Serum phospholipid fatty acids in severely injured patients on total parenteral nutrition with medium chain/long chain triglyceride emulsions. Ann Nutr Metab. 1995;39:251-260.

20. Daly JM, Lieberman MD, Goldfine J. Enteral nutrition with supplemental arginine, RNA, and omega-3 fatty acids in patients after operation: immunologic, metabolic, and clinical outcome. Surgery. 1992;112:56-67.
21. Kemen M, Senkal M, Homann H-H, Zumtobel V. Early postoperative enteral nutrition with arginine-omega-3-fatty acids and ribonucleic acid-supplemented diet versus placebo in cancer patients: an immunologic evaluation of Impact. Crit Care Med. 1995;23:652-659.

22. Kremer JM. n-3 Fatty acid supplements in rheumatoid arthritis. Am J Clin Nutr. 2000;71(suppl 1):349-351.

23. Segal-Isaacson CJ, Wylie-Rosett J. The cardiovascular effects of fish oils and omega-3 fatty acids. Heart Dis. 1999;1:149-154.

24. Takatsuka H, Takemoto J, Iwata N. Oral eicosapentaenoic acid for complications of bone marrow transplantation. Bone Marrow Transpl. 2001;28:769-774.

25. Ayala A, Deol ZK, Lehman DL, Herdon CD, Chaudry IH. Does endotoxin play a major role in inducing the depression of macrophage function during polymicrobial sepsis? Arch Surg. 1995; 130:1178-1184.

26. Grimminger F, Fuhrer D, Papavassilis C, et al. Influence of intravenous n-3 lipid supplementation on fatty acid profiles and lipid mediator generation in a patient with severe ulcerative colitis. Eur J Clin Invest. 1993;23:706-715.

27. Ling PR, Boyce P, Bistrian BR. Role of arachidonic acid in the regulation of the inflammatory response in TNF-alpha-treated rats. JPEN J Parenter Enteral Nutr. 1998;22:268-275.

28. Wachtler P, Hilger RA, Koenig W, et al. Influence of a preoperative enteral supplement on functional activities of peripheral leukocytes from patients with major surgery. Clin Nutr. 1995; 14:275-282.

29. Sanigorski AJ, Sinclair AJ, Hamazaki T. Platelet and aorta arachidonic and eicosapentaenoic acid levels and in vitro eicosanoid production in rats fed high-fat diets. Lipids. 1996;31:729735 .

30. Surette ME, Whelan J, Lu GP, Kinsella JE. Synthesis of arachidonic acid metabolites by Syrian hamster platelets and peritoneal cells. Lipids. 1993;28:1131-1134.

31. Heller H, Hoffmann G, Schuster KD, Schobersberger W. Endotoxemia affects lung vascular prostanoid metabolism in rabbits. J Appl Physiol. 1998;84:2200-2201.

32. Wolfram G. Diet in hyperlipidemia, hypertension and gout. Internist. 1995;36:1070-1076.

33. Elmadfa I, Stroh WS, Brandt K, Schlotzer E. Influence of a single parenteral application of a $10 \%$ fish oil emulsion on plasma fatty acid pattern and the function of thrombocytes in young adult men. Ann Nutr Metab. 1993;37:8-13.

34. Herrmann A, Döhla A, Hailer S, et al. Der Einfluss omega-3fettsäurehaltiger Emulsionen auf das Immunsystem gesunder Probanden unter Berücksichtigung der Fettsäurenzusammensetzung von Blutfetten und Zellmembranen. Aktuel Ernähr Med. 1996;21:63-64.

35. Ikehata A, Hiwatashi N, Kinouchi Y, et al. Effect of intravenously infused eicosapentaenoic acid on the leukotriene generation in patients with active Crohn's disease. Am J Clin Nutr. 1992;56:938-942.

36. Mayer K, Gokorsch S, Fegbeutel C. Parenteral nutrition with fish oil modulates cytokine response in patients with sepsis. Am J Respir Crit Care Med. 2003;167:1321-1328.

37. Morlion BJ, Torwesten E, Lessire A. The effect of parenteral fish oil on leucocyte membrane fatty acid composition and leukotriene-synthesizing capacity in postoperative trauma. Metabolism. 1996;45:1208-1213.

38. Roulet M, Frascarolo P, Pilet M, Chapuis G. Effects of intravenously infused fish oil on platelet fatty acid phospholipids composition and on platelet function in postoperative trauma. JPEN J Parenter Enteral Nutr. 1997;21:296-301.

39. Takagi K, Yamamori H, Furukawa K, Miyazaki M, Tashiro T. Perioperative supplementation of EPA reduces immunosuppression induced by postoperative chemoradiation therapy in patients with esophageal cancer. Nutrition. 2001;17:478-479.

40. Caughey GE, Mantzoris E, Gibson RA, Cleland LG. The effect of human tumor necrosis factor alpha and interleukin 1 beta production of diets enriched in n-3 fatty acids from vegetable and fish oil. Am J Clin Nutr. 1996;63:116-122.

41. Enders S, Ghorbani R, Kelly VE. The effect of dietary supplementation with n-3 polyunsaturated fatty acid on the synthesis of IL-1 and TNF alpha by mononuclear cells. $N$ Engl J Med. 1989;320:265-271. 